\title{
Rehabilitation of People With Chronic Spinal Cord Injury Using a Laparoscopically Implanted Neurostimulator: Impact on Mobility and Urinary, Anorectal, and Sexual Functions
}

\author{
Nucelio Lemos, MD, PhD ${ }^{1,2,3}$; Gustavo L. Fernandes, MD, $\mathrm{PhD}^{2,3,4}$; \\ Augusta M. Ribeiro, $\mathrm{MD}^{2,3}$; Priscila S. Maia-Lemos, CNP, PhD \\ Wellington Contiero, $\mathrm{PT}, \mathrm{MSc}^{2,3}$; Victor Croos-Bezerra, $\mathrm{PT}^{2,3}$; \\ George Tomlison, $\mathrm{PhD}^{5}$; Jean Faber, $\mathrm{PhD}^{6}$; Acary S.B. Oliveira, MD, $\mathrm{PhD}^{6, \mathrm{a}}$; \\ Manoel J.B.C. Girão, MD, PhD ${ }^{2, a}$
}

\begin{abstract}
Objectives: This study aimed to assess the impact of the laparoscopic implantation of neuromodulation electrodes (PossoverLION procedure) on mobility and on sexual, urinary, and anorectal functions of people with chronic spinal cord injury (SCI).

Material and Methods: Longitudinal analysis of 30 patients with chronic SCI (21 ASIA impairment scale (AIS) A, eight AIS B, and one AIS C) submitted to the Possover-LION procedure for bilateral neuromodulation of femoral, sciatic, and pudendal nerves. Assessments were performed before the surgical procedure and at 3, 6, and 12 months postoperatively. The primary outcome was evolution in walking, measured by the Walking Index for Spinal Cord Injury score, preoperatively and at 12 months. Secondary outcomes were changes in overall mobility (Mobility Assessment Tool for Evaluation of Rehabilitation score), urinary function and quality of life (Qualiveen questionnaire), and bowel (time for bowel emptying proceedings and Wexner's Fecal Incontinence Severity Index [FISI]) and sexual functions (International Index of Erectile Function for men and Female Sexual Function Index for women). Surgical time, intraoperative bleeding, and perioperative complications were also recorded.

Results: Qualitatively, 18 of 25 (72\%) patients with thoracic injury and 3 of $5(60 \%)$ patients with cervical injury managed to establish a walker-assisted gait at one-year follow-up $(p<0.0001)$. A total of $11(47.8 \%)$ have improved in their urinary incontinence $(p<0.0001)$, and seven (30.4\%) improved their enuresis $(p=0.0156)$. The FISI improved from a median of 9 points preoperatively to 5.5 at 12 months ( $p=0.0056)$. Of note, 20 of $28(71.4 \%)$ patients reported an improvement on genital sensitivity at 12 months postoperatively $(p<0.0001)$, but this was not reflected in sexual quality-of-life questionnaires.
\end{abstract}

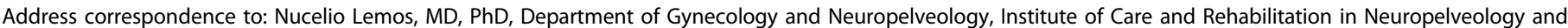
Gynecology, Rua Dr Bacelar, 227, Vila Clementino, CEP - 04026-000, São Paulo, SP, Brazil. Email: nucelio.lemos@increasing.com.br

1 Department of Obstetrics and Gynecology, Faculty of Medicine, University of Toronto, Toronto, Canada;

2 Department of Gynecology, Federal University of São Paulo, São Paulo, Brazil;

3 Department of Gynecology and Neuropelveology, Increasing-Institute of Care and Rehabilitation in Neuropelveology and Gynecology, São Paulo, Brazil;

4 Department of Obstetrics and Gynecology, Santa Casa de São Paulo School of Medical Sciences, São Paulo, Brazil; and

5 Institute of Health Policy, Management, and Evaluation, University of Toronto, Toronto, Canada; and

6 Department of Neurology and Neurosurgery, Federal University of São Paulo, São Paulo, Brazil

andicates equal contribution.

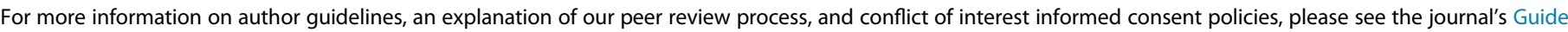
for Authors.

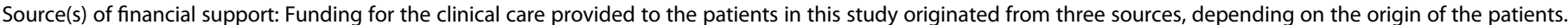

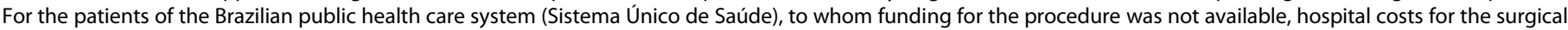

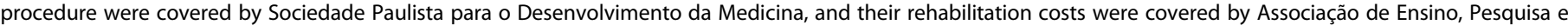

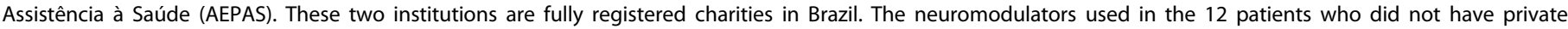

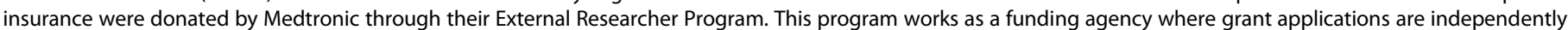

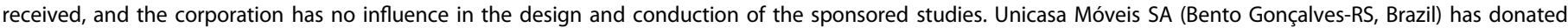

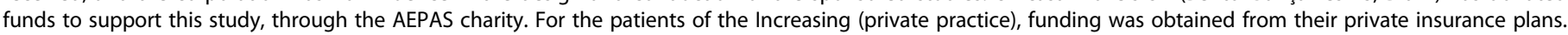

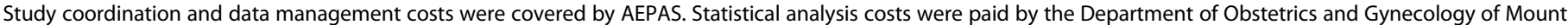

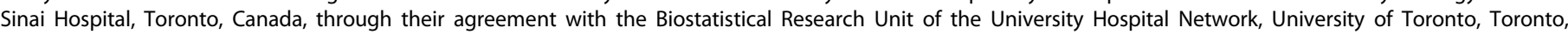
Canada. 
Conclusions: Patients experienced improved mobility and genital sensitivity and a reduction in the number of urinary and fecal incontinence episodes. By demonstrating reproducible outcomes and safety, this study helps establish the Possover-LION procedure as an addition to the therapeutic armamentarium for the rehabilitation of patients with chronic SCl.

Clinical Trial Registration: This study was registered at the WHO Clinical Trials Database through the Brazilian Registry of Clinical Trials-REBEC (Universal Tracking Number: U1111-1261-4428).

Keywords: Laparoscopy, LION procedure, neuropelveology, peripheral neuromodulation, spinal cord injury

Conflict of Interest: Nucelio Lemos has received speaker honoraria for one event promoted by Medtronic Inc. (Minneapolis, MN) in October 2019 and two events Promedon (Cordoba, Argentina) in March 2020 and April 2021. None of these events are related to this study. The remaining authors reported no conflict of interest.

\section{INTRODUCTION}

Spinal cord injury (SCl) is a disabling condition that causes sensorimotor loss and dysfunction of multiple organs. ${ }^{1}$ Although motor loss is the most evident consequence of $\mathrm{SCl}$, urinary and fecal incontinence, genital desensitization, loss of tactile sensitivity, and even cognitive deficits have an even worse impact on the social, psychologic, and physical wellbeing of individuals with this condition. ${ }^{2,3}$ Therefore, innovative solutions are needed to approach all these dysfunctions. To achieve that goal, different efforts have been made to improve the rehabilitation process through assistive technologies, neuroprosthetic devices, and other new therapies. ${ }^{4,5}$ This study focuses on one of these innovations.

Initially described by Possover et $\mathrm{al}^{6-11}$ as a recovery procedure for patients after explantation of a Finetech-Brindley device (Finetech Medical Ltd, Welwyn Garden City, UK), ${ }^{6}$ the laparoscopic implantation of neuromodulation (LION) electrodes has been further adapted for reestablishing gait and improving bladder, bowel, and sexual function of patients with $\mathrm{SCl}^{7-11}$

Based on the good initial results in patients with chronic $\mathrm{SCl}_{1}^{7-11}$ the Possover-LION procedure became approved and available in Brazil, being considered a level $1 \mathrm{C}$ evidence for promoting locomotion in patients with $\mathrm{SCl}$, and insurance companies started to reimburse the surgical procedure and its related rehabilitation process. In December 2013, we started offering those procedures and established a multiprofessional rehabilitation team specialized in Neuropelveology.

This study aimed to document changes in mobility and in sexual, urinary, and anorectal functions of people with chronic $\mathrm{SCl}$ over the first postoperative year of the Possover-LION procedure, adding a more rigorous, objective, and prospective results analysis to the literature, because all evidence currently available is based on narrative case series.

\section{MATERIALS AND METHODS}

This is a longitudinal analysis with repeated measures of our series of patients with chronic SCl submitted to the Possover-LION procedure for bilateral neuromodulation of femoral, sciatic, and pudendal nerves, operated between December 2013 and June 2019. Patients originated from the Division of Pelvic Neurodysfunctions of the Department of Gynecology of the Federal University of São Paulo and from the Increasing-Institute for Care and Rehabilitation in Neuropelveology and Gynecology, São Paulo, Brazil.
Patients were offered the Possover-LION procedure if they sustained a chronic $\mathrm{SCl}$ and their neurological level remained stable after one year or more of traditional rehabilitation. Patients with cervical lesions were only offered the procedure if their lesion was incomplete and if they had a grade $\geq 2$ triceps muscle power and their hand function was good enough to allow the operation of the neuromodulator's remote control. Patients with thoracic injuries were offered the procedure regardless of whether they had sustained a complete or incomplete lesion.

Clinical outcome data for all patients were systematically collected in a specific data collection form and extracted to an Excel $^{\oplus}$ (version 16.29.1; Microsoft ${ }^{\oplus}$ Inc, Redmond, WA) worksheet.

\section{Interventions}

Preoperative Rehabilitation Protocol

The preoperative rehabilitation protocol was focused on the stretching of shortened muscles, in preparation for postoperative rehabilitation, and training of transfers and other activities of daily living (ADLs), which must be modified to minimize the risk of postoperative electrode dislocation. Once the patient and the caregivers had learned all the adapted transfers and ADLs, the surgical procedure was booked. This adaptation period lasted between one and six months, depending on the patient's physical condition and patient and caregiver learning speed.

Patients also underwent a cardiologic evaluation to assess their conditioning to the high intensity postoperative rehabilitation. From the sixth patient onward, they also underwent a preoperative microbiological assessment, with characterization of their oropharyngeal, nasopharyngeal, anorectal, urinary, and skin flora (inguinal, axillar, hands, and abdominal samples), to tailor their surgical antibiotic prophylaxis.

\section{Surgical Procedure}

Procedures were performed according to the technique proposed by Possover et al. ${ }^{6}$ The laparoscopic neuronavigation (LANN) technique $^{11}$ was used to expose the intrapelvic portion of the superior gluteal, sciatic, pudendal, and femoral nerves.

Either Medtronic ${ }^{\circledR} \quad$ (Restore Ultra $^{\circledast}$ pulse generator; Pisces ${ }^{\circledR}$ quadripolar electrodes [Medtronic, Dublin, Ireland]) or Boston Scientific $^{\circledast}$ (Precision Spectre ${ }^{\circledR}$ pulse generator; Linear ${ }^{\circledR}$ octapolar electrodes [Boston Scientific, Marlborough, MA]) neuromodulation systems were used, according to the availability (insurance plan, hospital contract, donations, etc). Boston Scientific ${ }^{\circledR}$ systems were preferred for having four eight-pole channels, as opposed to the two eight-pole channels on the Medtronic ${ }^{\circledR}$ pulse generator. The four-channel/32-pole configuration provides faster intraoperative 
assembly with less overall volume of implanted material because no bifurcated connection is needed. It also allows for more accurate stimulation because of the larger number of contact poles in the same electrode stimulation area (eight vs four).

One electrode was placed juxta-laterally to each femoral nerve and fixed to the fascia transversalis. One electrode was used at each side for both the sciatic and pudendal nerves, with half of the poles (two for the Medtronic ${ }^{\circledR}$ system and four for the Boston Scientific ${ }^{\circledast}$ system) into the pudendal canal and half of the poles over the ventral surface of the sciatic and superior gluteal nerves. Figure 1 illustrates the electrodes positioning-an animated graphic demonstration also is available in Supplementary Data Video S1.

\section{Neuromodulator Programming and Rehabilitation Protocol}

Neuromodulation and neurostimulation protocols entail the creation of situation-specific program groups, to be used in specific daily life situations.

Basic programming includes a baseline low-frequency protocol $(5-20 \mathrm{~Hz})$ that is kept continuously active throughout the day to control bladder overactivity, promote a baseline tonus, and control spasticity on inferior limb muscles and promote neuroplasticity; individual mid-frequency $(20-40 \mathrm{~Hz})$ protocols that are activated on demand to promote intermittent contractions of quadriceps, deep gluteal, and pelvic floor muscles for training/physiotherapy purposes; and a continuous stimulation protocol for orthostatism and gait training.

The patient uses a remote control to select and activate the programs as they are needed.

Other programs were sporadically created under indication or patient request to facilitate penile erection and ejaculation, optimize lower limb edema reabsorption with intermittent calf contractions, and facilitate specific transfer demands, among others. Because these were created on demand and were extremely variable among patients, there were not enough data for the analysis of these specific stimulation protocols, and this will be the object of a future study.

Table 1 describes the parameters and target nerve for each of the main programs, and Supplementary Data Video S1 illustrates those programs in action. The lowest frequency to induce harmonic contraction (with no tremors/vibration) was used for the programs that stimulate the femoral, sciatic, and superior gluteal nerves, because higher frequencies induce quick-setting muscle fatiguefrequencies below 40 to $50 \mathrm{~Hz}$ are known to preferentially activate type I, slow-twitch muscle fibers that are more resistant to muscle fatigue, ${ }^{12,13}$ whereas higher frequencies recruit more type lla and type Ilb fast-twitch muscle fibers that are easily fatigued. For training of the pelvic floor muscles, we use the same approach, considering lower frequencies $(30 \mathrm{~Hz})$ for the training of slow fibers and higher frequencies $(50 \mathrm{~Hz})$ for fast fibers. Pudendal neuromodulation at 10 to 15 $\mathrm{Hz}$ is also used to improve bladder compliance and reduce neurogenic detrusor overactivity. ${ }^{14,15}$

\section{Postoperative Rehabilitation Protocol}

All patients underwent an intensive rehabilitation protocol to learn how to use the movements generated by the neuromodulator to enhance their rehabilitation and ADLs. This protocol comprised 15 to 20 weekly hours of multidisciplinary care, including neurofunctional physiotherapy and aquatic therapy (eight to ten hours/week), cardiorespiratory physiotherapy (two hours/week), occupational therapy (one to two hours/week), pelvic physiotherapy (one to two hours/week), monthly nutritional follow-up, multidisciplinary neuromodulation sessions for adjustments to the neuromodulation settings (as needed), analgesic physiotherapy (as needed), quarterly cardiologic assessment, and monthly physiatrist assessment/follow-up.

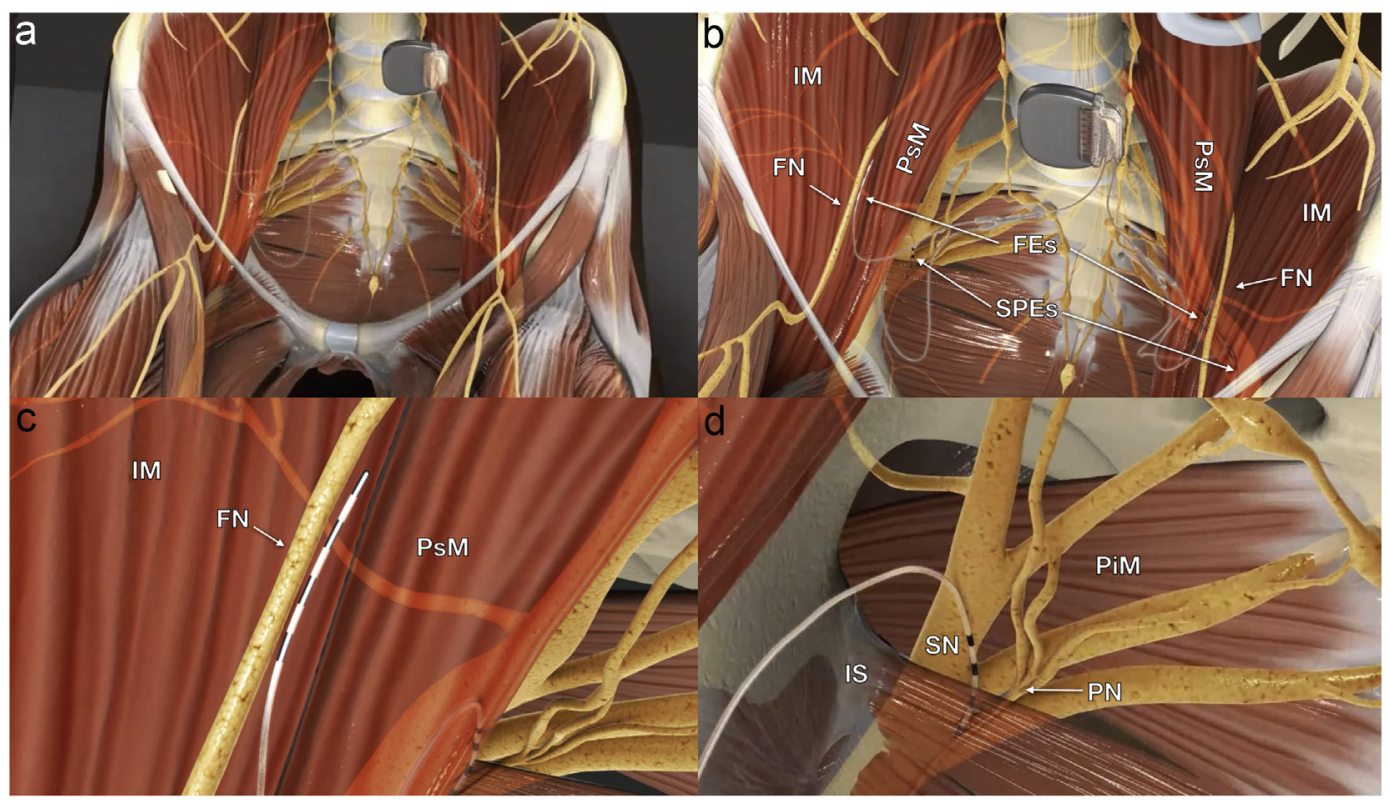

Figure 1. Electrode positioning. a. Panoramic view of the system; the pulse generator is implanted into a paraumbilical subcutaneous pocket, and the electrodes run retroperitoneally down to the intrapelvic portions of the femoral (FN), sciatic (SN), and pudendal nerves (PN) bilaterally. b. Panoramic view of femoral electrodes (FEs) and sciatic and pudendal electrodes (SPEs) positioning. c. Detailed view of the right femoral electrode over the nerve between the iliac (IM) and the psoas (PsM) muscles. d. Detailed view of the right sacral electrodes placed with half of its poles in the Alcock's canal over the PN and the remaining poles over the SN. IS, ischial spine; PiM, piriformis muscle. [Color figure can be viewed at www.neuromodulationjournal.org] 
During the first two postoperative weeks, patients are instructed to continuously use program $A$ and activate programs B-F at home for two minutes, three times daily, to start the recruitment of muscle fibers. After this initial period, they start the rehabilitation protocol in the clinic. For three months, because of the risk of electrode displacement, strengthening exercises are performed in stable positions only (lying down, sitting, or at the stand table).

In the fourth postoperative month, they start progressively practicing standing up from the wheelchair to the parallel bar and weight transfer from one leg to another in the standing position on the parallel bars, and then, they start taking steps. The same process is then trained with the walker, and then, the walker-assisted movements are incorporated into their ADLs at home and in the community.

During this period, patients also practice how to integrate neuromodulation-generated movements into their ADLs to facilitate transfers and position changes, using pelvic floor exercises to defer urinary urgency episodes (in patients with incomplete sensory injury), etc.

\section{Outcome Measures}

Assessments were performed just before the surgical procedure (T0) and at three, six, and 12 months postoperatively (respectively, $\mathrm{T} 3, \mathrm{~T} 6$, and $\mathrm{T} 12)$.

Mobility

The primary study outcome was the evolution of the Walking Index for Spinal Cord Injury (WISCI II), ${ }^{16}$ a standardized scale that classifies the individual's walking ability in 21 levels, from 0 to 20 (Table 2), from the preoperative to the 12-month assessment.

Because the WISCI II only assesses gait, we also have developed the Mobility Assessment Tool for Evaluation of Rehabilitation (MAsTER), which assesses overall mobility based on the mobility landmarks identified by our team as those with the most impact on patients' ADLs (Table 3).

\section{Urinary Function}

Urinary incontinence was assessed using a 5-point scale: 0 , no incontinence; 1, less than one incontinence episode per week; 2 , one to six incontinence episodes per week; 3, one to two incontinence episodes per day; and 4, more than two incontinence episodes per day.

Nocturnal enuresis was categorized using a 4-point scale: 0 , no noticeable urinary loss during sleep hours; 1 , nocturnal enuresis controlled with diapers or condom catheters, with no impact on sleep pattern; 2, nocturnal enuresis controlled with diapers, but disturbing a patient's sleep quality; and 3, nocturnal enuresis not controlled with diapers, with severe impact on hygiene and sleep quality.

Impact of urinary incontinence on quality of life was measured with a Brazilian Portuguese validated version of the Qualiveen questionnaire. $^{17}$

Urodynamic maximum cystometric capacity, bladder compliance, and volume at the first detrusor overactivity were measured at T0, T6, and T12. First sensation was not reported, because most participants did not report any bladder sensation, except for occasional mild dysreflexia (chills, piloerection, and tachycardia) triggered by bladder filling. 
Table 2. The WISCI II Score.

\section{Level Description}

$0 \quad$ Client is unable to stand and/or participate in assisted walking.

1 Ambulates in parallel bars, with braces and physical assistance of two persons, $<10 \mathrm{~m}$.

2 Ambulates in parallel bars, with braces and physical assistance of two persons, $10 \mathrm{~m}$.

3 Ambulates in parallel bars, with braces and physical assistance of one person, $10 \mathrm{~m}$.

$4 \quad$ Ambulates in parallel bars, no braces and physical assistance of one person, $10 \mathrm{~m}$.

Ambulates in parallel bars, with braces and no physical assistance, $10 \mathrm{~m}$.

6 Ambulates with walker, with braces and physical assistance of one person, $10 \mathrm{~m}$.

7 Ambulates with two crutches, with braces and physical assistance of one person, $10 \mathrm{~m}$.

8 Ambulates with walker, no braces and physical assistance of one person, $10 \mathrm{~m}$

9 Ambulates with walker, with braces and no physical assistance, $10 \mathrm{~m}$

10 Ambulates with one cane/crutch, with braces and physical assistance of one person, $10 \mathrm{~m}$.

11 Ambulates with two crutches, no braces and physical assistance of one person, $10 \mathrm{~m}$.

12 Ambulates with two crutches, with braces and no physical assistance, $10 \mathrm{~m}$.

13 Ambulates with walker, no braces, and no physical assistance, 10 $\mathrm{m}$.

14 Ambulates with one cane/crutch, no braces and physical assistance of one person, $10 \mathrm{~m}$.

15 Ambulates with one cane/crutch, with braces and no physical assistance, $10 \mathrm{~m}$.

16 Ambulates with two crutches, no braces, and no physical assistance, $10 \mathrm{~m}$.

17 Ambulates with no devices, no braces and physical assistance of one person, $10 \mathrm{~m}$.

18 Ambulates with no devices, with braces and no physical assistance, $10 \mathrm{~m}$

19 Ambulates with one cane/crutch, no braces, and no physical assistance, $10 \mathrm{~m}$.

20 Ambulates with no devices, no braces, and no physical assistance, $10 \mathrm{~m}$

\section{Bowel Function}

Time to complete bowel proceedings was categorized as normal ( $<30$ minutes), long (30-60 minutes), or very long (>60 minutes). The Fecal Incontinence Severity Index (FISI) ${ }^{18}$ was used to quantify anal incontinence.

\section{Sexual Function}

Sexual function was assessed at each visit with the International Index of Erectile Function (IIEF) ${ }^{19}$ for men and the Female Sexual Function Index $(\mathrm{FSFI})^{20}$ for women. Patients also were asked about genital sensitivity during coitus, masturbation, and other sexual activities; at each assessment, they were asked if they felt that their sensitivity was improved, unchanged, or worsened since their last follow-up.

\section{Surgical Morbidity}

Surgical morbidity was assessed by means of surgical time, measured from first incision to the placement of the last dressing;
Table 3. MAsTER Score.

Score Description

$0 \quad$ Totally dependent on others for positional changes and transfers.

1 Able to roll on bed; dependent on two helpers for sitting and transfers.

2 Able to independently roll on bed and sit, and to roll the wheelchair; dependent on one helper for transfers.

3 Independent for rolling, sitting, rolling the wheelchair, and transferring with transfer board.

$4 \quad$ Independent for rolling, sitting, rolling the wheelchair, and transferring between uneven surfaces with $>20 \mathrm{~cm}$ difference without a transfer board.

Able to stand up on stall bars.

Able to stand up on parallel bars.

Therapeutic gait on parallel bars.

Therapeutic gait on walker.

Functional gait with walker at home.

Functional gait with walker on community with supervision.

Functional gait with walker on community without supervision.

Therapeutic gait with crutches.

Functional gait with 4 points cane.

Functional gait with 1 point cane.

Normal gait.

intraoperative bleeding quantified from the suction canister (no sponges were used for bleeding control); and perioperative complications, which were recorded and described as individual events and categorized according to the Clavien-Dindo classification. ${ }^{21}$

\section{Statistical Analysis}

Data analysis was performed using $\mathrm{R}$ (version 3.6.2; R Core Team, 2019, The R Foundation for Statistical Computing, Vienna, Austria). Patient demographics and baseline characteristics were summarized using means with SDs for continuous variables and counts and percentages for categorical variables. Outcome measures were similarly summarized at baseline and at three, six, and 12 months, and individual patient trajectories and medians were plotted over time. For continuous outcome measures and the ordinal WISCI II and MAsTER scales, the Friedman test was used to assess withinperson differences in the median overall assessments from baseline to 12 months. For the 3-to-5-point ordinal outcomes (incontinence, nocturnal enuresis, time for bowel proceedings, and perineal sensitivity), 12-month values were compared with baseline outcomes and classified as unchanged, improved, or worsened. Counts in these last two categories were used in an exact binomial test of the hypothesis that there was more improvement than worsening.

A two-sided $p$-value $<0.05$ was taken as indicating statistical significance.

\section{Power and Sample Size}

The minimal clinically significant difference (MCID) for the WISCl II scale is 2 points. ${ }^{22}$ Considering a one-tailed significance threshold (a) of $5 \%$ and power $(\beta)$ of $80 \%$ and a magnitude of change of 2 points for the WISCI II score, the minimal necessary sample size would be 25 participants (assuming a standard deviation of 5.5, according to the worst case of our findings).

Using the same parameters, our sample size provides a power $(\beta)$ of $90 \%$ to detect a difference of 2 points on the WISCI II score and 
carries a risk of $5 \%$ of false-positive results (a) for this same parameter, calculated through the relation $n=\left(\frac{z_{a}+z_{\beta}}{\left|\mu_{0}-\mu_{1}\right|} \sigma\right)^{2}$, where $\mu_{0}=0$ is the reference (null) mean and $\mu_{1}$ the true mean for statistic effect.

Power calculations also were performed to assess the ability of our sample size to detect an MCID to our secondary outcome measures, also considering a two-tailed significance threshold (a) of $5 \%$ and power $(\beta)$ of $80 \%$ and the following magnitudes of changes:

- For the MAsTER score, a 1-point difference is already clinically important; a minimal sample size of 35 would be necessary to detect that difference (assuming a standard deviation of 4.05, according to our findings).

- For the Qualiveen, an anchor-based approach, the MCID is $-0.5,{ }^{23}$ requiring a minimal sample size of 70 participants (assuming a standard deviation of 14, according to our findings).

- For the FISI, the MCID is -3 points, ${ }^{24}$ requiring a minimal sample size of 84 participants (assuming a standard deviation of 6.25 , according to our findings).

- For the FSFI questionnaire, the MCID is 1 point, $^{25}$ requiring a minimal sample size of 45 participants (assuming a standard deviation of 6.5, according to reference).

- For the IIEF questionnaire, the MCID is 1 point, $^{26}$ requiring a minimal sample size of 60 participants (assuming a standard deviation of 5.95, according to reference).

\section{Ethical Aspects}

All patients signed consent authorizing the use of their clinical data for research and educational purposes. This research protocol was approved by the Research Ethics Review Board of the Federal University of São Paulo (Protocol Number 1.681.992). This study was registered at the WHO Clinical Trials Database through the Brazilian Registry of Clinical Trials-REBEC (Universal Tracking Number: U1111-1261-4428).

\section{RESULTS}

A total of 30 patients (seven women, 23 men) underwent the Possover-LION procedure for rehabilitation of $\mathrm{SCl}$ within the inclusion period. Patient demographics and $\mathrm{SCl}$ classifications are displayed in Table 4. All patients sustained chronic SCl with no improvement on the neurological sequelae for at least one year before the Possover-LION procedure.

The most significant impact was on mobility measures. Median WISCI II score (primary outcome) evolved from a median 0 to median 5 ( $p<0.0001$; Friedman test; Fig. 2$)$, and the median MAsTER score increased from four to seven $(p<0.0001$; Friedman test; Fig. 2). The thin lines on Figure 2 show how $100 \%$ of the patients improved mobility and all but two patients managed to initiate gait training. Qualitatively, 18 of 25 (72\%) patients with thoracic injury and three of five (60\%) patients with cervical injury managed to establish independent walker-assisted gait (WISCI ॥ score $\geq 7$ ), using only supramaleolar ankle-foot orthosis to stabilize their ankles (Fig. 2).

A total of 23 patients provided information on urinary incontinence episodes at 0 and 12 months. Of these, 11 (47.8\%) have improved their urinary incontinence category, and 12 (52.2\%) were unchanged; none has worsened ( $p<0.0001$; Fig. 3, Supplementary Data Tables S1-S4). Nocturnal enuresis was unchanged in 16 patients $(69.6 \%)$ and improved in seven $(30.4 \%)$ patients $(p=0.0156$; Fig. 3; Supplementary Data Tables S5-S10). Urodynamics data for both preoperative and six- and 12-month postoperative tests were available. No statistically significant changes were noticed on maximum cystometric capacity, volume at first detrusor overactivity, or bladder compliance (Fig. 3, Supplementary Data Tables S11-S13, and Supplementary Data Fig. S1-S3). From a quality-of-life standpoint, no significant changes were observed in any of the Qualiveen fields (Supplementary Data Tables S14-S23 and Supplementary Data Figs. S4-S14). The dose of anticholinergics remained stable in the follow-up period (Supplementary Data Tables S24-S26 and Supplementary Data Fig. S15).

The positive impact on bowel function was more evident. FISI improved from a median of 9 points preoperatively to 5.5 at 12 months ( $p=0.0056$; Fig. 4, Supplementary Data Tables S27 and S28 and Supplementary Data Fig. S16). Data on time to perform bowel proceedings at T0 and T12 were available for 20 patients. Preoperatively, $52 \%(12 / 23)$ of the patients needed $>30$ minutes to perform their bowel proceedings; at two month follow-up, 65\% $(13 / 20)$ of the patients were taking $<30$ minutes for their bowel routine ( $p=0.025$; exact binomial test; Fig. 4 , Supplementary Data Tables S29-S31).

With regard to sexual function, the pre- and postoperative IIEF were filled by 15 of $24(62.5 \%)$ men and the FSFI by five of seven women. No statistically significant score changes were observed from T0 to T12 (Fig. 5, Supplementary Data Table S32-S34, and Supplementary Data Fig. S17). However, 20 of 28 (71.4\%) patients reported an improvement on genital sensitivity at 12 months postoperatively; the rest were unchanged ( $p<0.0001$; Fig. 5, Supplementary Data Tables S35-S39).

Mean surgical time was 230 minutes $( \pm 74 ; 133-420)$, and no intraoperative complications were registered. Intraoperative bleeding was below $100 \mathrm{~mL}$ in all cases. The most frequent postoperative complication was electrode displacement, which happened in three $(10 \%)$ cases and required a reintervention for electrode repositioning (Clavien-Dindo grade IIIb); the displaced electrodes were left femoral nerve in the first case, right femoral nerve in the second case, and both femoral and pudendal nerves on the left side in the third case. The three displacements occurred within the first three months after the procedure and in situations where the movements that should be avoided were inadvertently performed. Another complication observed was one case $(3.3 \%)$ of infection of the neuromodulator, noticed at the seventh postoperative week, in the fifth case in this series; several antibiotic courses were attempted with no success, resulting in the explantation (Clavien-Dindo grade $\mathrm{IIlb}$ ) of the neuromodulator at five months postoperatively, with no further complications; in fact, despite the explantation, the patient maintained some of the functional gains observed in the short rehabilitation period and improved his ASIA impairment scale (AIS) grade from B to C. After that index case, we included an infectious diseases specialist in our team for preoperative assessment and tailoring of the antimicrobial prophylaxis, based on the bacterial flora of the subject. We have since not documented any other case of infection, setting our infection rate at $3.3 \%$ by the time this manuscript was submitted.

No discomfort or pain was reported by any patient, and no episodes of dysreflexia were observed as a result of nerve stimulation. 
Table 4. Patient Demographics and SCI Characteristics.

\begin{tabular}{|c|c|c|c|c|c|c|}
\hline Age & Sex & SCl level & Neurologic level & AIS & Etiology & Time since injury $(y)$ \\
\hline 30.9 & M & $\mathrm{T} 1$ & $\mathrm{~T} 1$ & $A$ & Motor vehicle accident & 11 \\
\hline 25.0 & M & C5 & C5 & B & Snowboard accident & 5 \\
\hline 35.9 & $\mathrm{~F}$ & $\mathrm{~T} 10$ & T6 & A & Motor vehicle accident & 4 \\
\hline 29.7 & M & C6 & $\mathrm{C} 5$ & A & Motorcycle accident & 2 \\
\hline 32.8 & M & C6 & C6 & B & Motor vehicle accident & 8 \\
\hline 51.7 & M & $\mathrm{T} 7$ & $\mathrm{~T} 7$ & A & Hit by a truck as cyclist & 4 \\
\hline 28.8 & $\mathrm{~F}$ & T4 & T5 & A & Hit by a car as pedestrian & 28 \\
\hline 49.9 & M & $\mathrm{T7}$ & $\mathrm{T7}$ & A & Fall from a tree & 6 \\
\hline 50.4 & M & T5 & T4 & A & Gunshot & 20 \\
\hline 43.2 & M & $\mathrm{T} 10$ & $\mathrm{~T} 7$ & A & Motorcycle accident & 3 \\
\hline 25.5 & $\mathrm{~F}$ & $\mathrm{~T} 10$ & T9 & B & Gunshot & 3 \\
\hline 45.0 & $\mathrm{~F}$ & $\mathrm{T7}$ & $\mathrm{T7}$ & B & Spinal surgery trauma & 2 \\
\hline 30.4 & M & T5 & T4 & A & Motorcycle accident & 3 \\
\hline 30.1 & M & $C 7$ & $\mathrm{C7}$ & C & Spinal surgery trauma & 8 \\
\hline 33.0 & $\mathrm{~F}$ & $\mathrm{~T} 7$ & $\mathrm{T7}$ & A & Motor vehicle accident & 14 \\
\hline 36.6 & F & $\mathrm{T} 4$ & $\mathrm{~T} 1$ & A & Motor vehicle accident & 3 \\
\hline 40.9 & $\mathrm{~F}$ & $\mathrm{T7}$ & $\mathrm{T} 7$ & A & Gunshot & 13 \\
\hline 25.5 & M & $\mathrm{T} 2$ & $\mathrm{~T} 2$ & A & Gunshot & 3 \\
\hline 28.3 & M & $\mathrm{T} 10$ & $\mathrm{~T} 10$ & A & Fall from rooftop & 1 \\
\hline 28.5 & M & $\mathrm{T} 4$ & T4 & A & Motorcycle accident & 7 \\
\hline 41.2 & M & T5 & T5 & A & Motorcycle accident & 19 \\
\hline 35.5 & M & $\mathrm{T} 10$ & T9 & A & Motorcycle accident & 9 \\
\hline 35.5 & M & $\mathrm{T7}$ & T7 & A & Motor vehicle accident & 6 \\
\hline 50.3 & M & T4 & T4 & A & Spinal stroke & 3 \\
\hline 33.5 & M & C6 & C6 & B & Motorcycle accident & 14 \\
\hline 23.1 & M & $\mathrm{T} 12$ & $\mathrm{~T} 11$ & A & Motorcycle accident & 2 \\
\hline 32.0 & M & T6 & T6 & A & Motorcycle accident & 3 \\
\hline 40.4 & M & T8 & T8 & $A$ & Motorcycle accident & 4 \\
\hline 34.9 & M & $\mathrm{C} 6$ & $\mathrm{~T} 1$ & B & Shallow water diving & 6 \\
\hline 35.0 & M & $\mathrm{T} 1$ & T5 & B & Gunshot & 6 \\
\hline $35.5( \pm 8.1)$ & $\begin{array}{l}7 \mathrm{~F}(23.3 \%) \\
\quad 23 \mathrm{M}(76.7 \%)\end{array}$ & $\begin{array}{l}5 \mathrm{C}(16.7 \%) \\
13 \mathrm{~T} 1-\mathrm{T5}(43.3 \%) \\
12 \mathrm{~T} 6-\mathrm{T} 12(40 \%)\end{array}$ & $\begin{array}{l}5 \mathrm{C}(16.7 \%) \\
11 \mathrm{~T} 1-\mathrm{T} 5(36.7 \%) \\
14 \mathrm{~T} 6-\mathrm{T} 12(46.6 \%)\end{array}$ & $\begin{array}{l}21 \text { A }(70 \%) \\
8 \text { B }(26.7 \%) \\
1 \text { C }(3.3 \%)\end{array}$ & $\begin{array}{l}18 \text { motor vehicle accident (60\%) } \\
5 \text { gunshot }(6.7 \%) \\
4 \text { sports/falls }(13.3 \%) \\
3 \text { stroke/surgery }(10 \%)\end{array}$ & $7.3( \pm 6.3)$ \\
\hline
\end{tabular}

Age, patient age at the time of device implant; SCI level, orthopedic level of trauma/stroke; neurologic level, neurologic level at the time of device implantation; AIS, ASIA impairment scale at the time of device implantation; etiology, etiology of SCl; time since injury, time (in years) between the injury and device implantation.

\section{DISCUSSION}

This study reports the results of a rehabilitation strategy based on the use of neuromodulation and functional electrical stimulation (FES) generated by a neurostimulator implanted using the LANN technique-the Possover-LION procedure. ${ }^{6,11}$ The mobility outcomes of this procedure, associated with a rehabilitation protocol that is based on the use of the FES-induced movements generated by the neuromodulator, are quite remarkable: all of the patients were able to extend their knees on the first postoperative day, and at one-year follow-up, only two of the 30 patients were not able to perform gait training using only ankle-foot orthotics, which fit into the patients' shoes and allow for the knee extension to be used on their day-to-day mobility. We also have observed improvements in bowel function, as well as some positive impact on urinary and sexual functions.

The implantation of neuroprosthesis for recovery of locomotion of people with $\mathrm{SCl}$ has been attempted in animal and human participants since the late sixties. ${ }^{27}$ In the early seventies, Brindley ${ }^{28}$ published the successful induction of micturition through sacral anterior root stimulation (SARS), using a device originally designed to improve walking.

Ironically, the laparoscopic implantation of neurostimulators started as a correction procedure for patients with damaged SARS electrodes, in whom a new posterior approach was not feasible. ${ }^{6}$ At that time, Possover et $\mathrm{al}^{11}$ had developed the LANN technique to identify and expose intrapelvic portions of the sacral plexus, aiming to reduce postoperative functional morbidity during radical pelvic procedures. The LANN technique was then successfully used to implant Finetech-Brindley ${ }^{\circledR}$ electrodes in patients previously submitted to a posterior (spinal) approach for the SARS procedure and whose electrodes had to be explanted because of material failure or infection. ${ }^{6}$ The good results with the recovery procedure encouraged Possover et al ${ }^{6}$ to develop new strategies for the laparoscopic implantation of neuroprosthesis, stimulating the pudendal, sciatic, and femoral nerves-a procedure now known as the Possover-LION procedure.

Other strategies for using epidural stimulation on the rehabilitation of people with $\mathrm{SCl}$ have shown some promising results. 

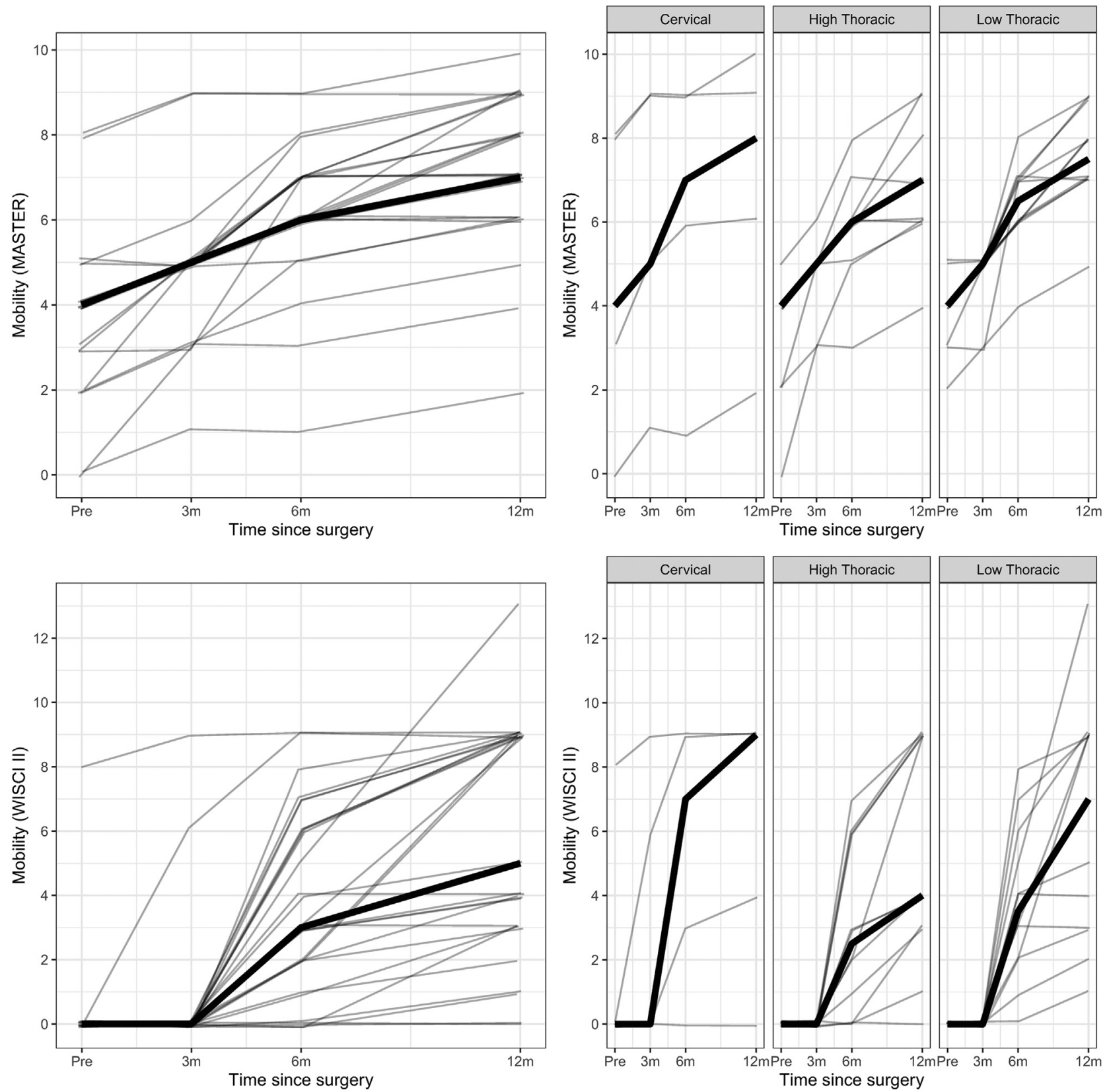

Figure 2. Mobility assessment at four time points (presurgery and three, six, and 12 months after surgery). The top row shows individual trajectories (thin lines) and the median (thick line) on the MAsTER mobility score, and the bottom row shows individual trajectories (thin lines) and the median scores (thick line) on the WISCI II. The left panel in each row shows all patients and the right panel groups patients according to the level of the SCl. All patients have improved their mobility status (increased their MAsTER score) and all but two patients were able to engage in gait training (WISCI II $\geq 7$ ).

These results are mostly based on neuroplasticity and facilitation of remaining neural pathways because direct spinal cord stimulation does not work like FES and cannot by itself induce a coordinated and effective movement. Therefore, epidural stimulation facilitates the process of rehabilitation through neuroplasticity and synaptic facilitation, but not through producing stimulator-generated movements. ${ }^{29-35}$

Our rehabilitation strategy is based on the stimulation of peripheral nerves, which is "the most established, clinically accessible, and well-tested form of neuromodulation for $\mathrm{SCl}^{.1{ }^{36}}$ This strategy allows for extension of the knees immediately after surgery, and patients can start with strengthening exercises on postoperative day two. Three months after the surgery, most of our patients with paraplegia were already able to stand up and start gait training on parallel bars, even if they sustained a motor-complete injury, which is the case for 29 of the 30 patients in our sample. As core control improves, patients start introducing those movements into their ADLs, becoming less dependent on the wheelchair for walking short distances, especially at home. They also become progressively less dependent on adapted environments-for example, being able to stand up and reach for objects from shelves or walk into a nonadapted shower and sit on a standard chair for showering. All these gains are very predictable, as 

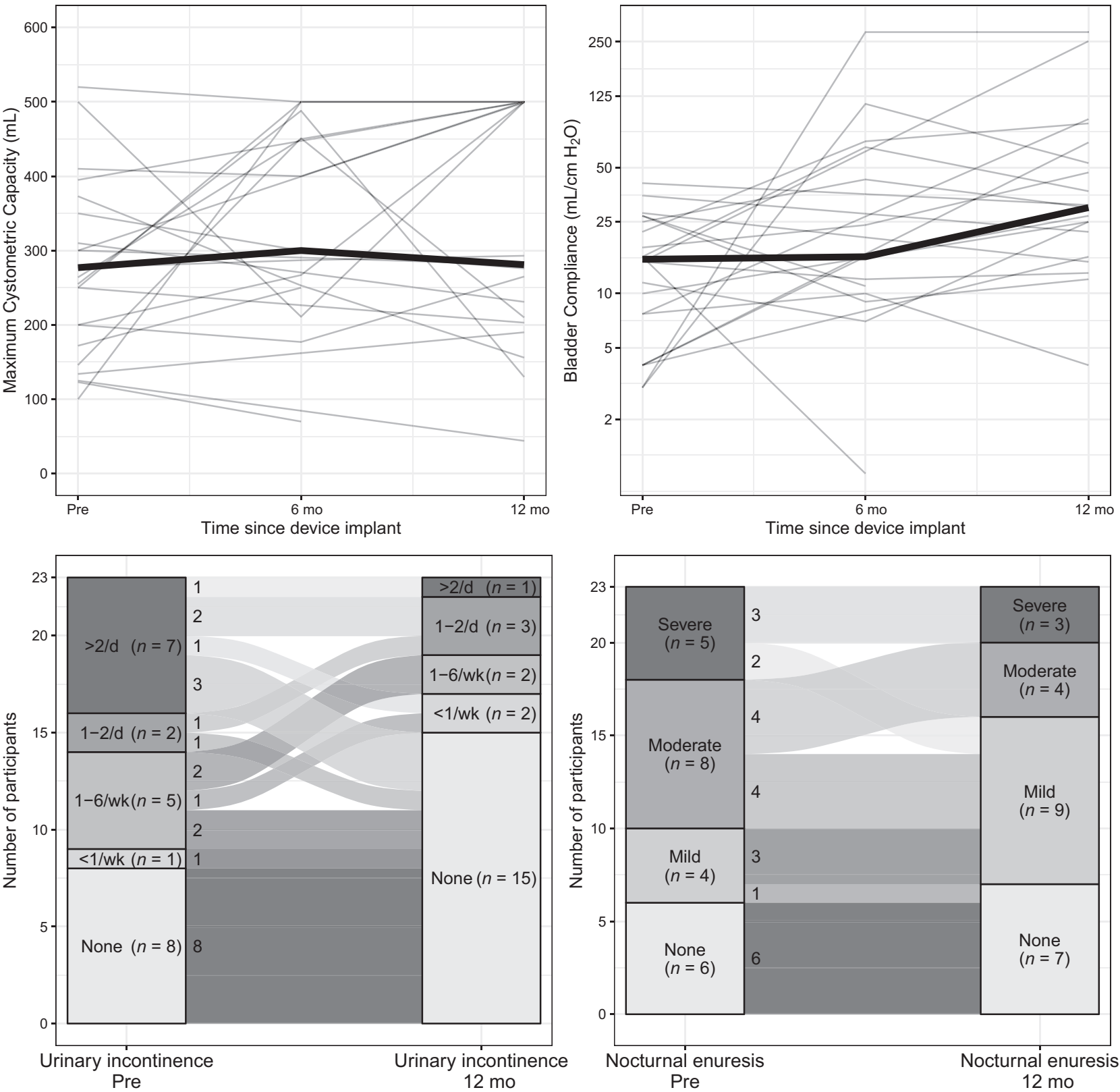

Figure 3. Urinary function. The top left panel shows individual trajectories and the median of maximum cystometric capacity $(\mathrm{mL})$ at three time points (presurgery and six and 12 months after surgery.) The top right panel shows individual trajectories and the median for bladder compliance; the $y$-axis is labeled with log-scaled values in $\mathrm{mL} / \mathrm{cm} \mathrm{H}_{2} \mathrm{O}$. The bottom left panel shows the distribution of patients across five urinary incontinence groups presurgery and at 12 months after surgery. The area of each component of a bar is proportional to the number of patients in the corresponding group (the $n$ in the bars reflects total number of participants in each group, and the number in the shaded area reflects the number of individuals at each trajectory). Each shaded curve connecting the two sets of bars corresponds to a different pattern of presurgery and 12-month outcomes. The bottom right panel presents data on nocturnal enuresis in the same way.

demonstrated on the curves for individual patients on the plots of MAsTER and WISCI II over time-all patients in this study have improved their MAsTER scores, and all but two have improved their WISCI II scores. In other words, only two of 29 motor-complete (AIS A or $\mathrm{B}$ ) patients with $\mathrm{SCl}$ were not able to engage in gait training with this treatment. The main reason for these remarkable mobility gains is that our mobility strategy is based on stimulator-induced movements instead of being totally dependent on neuroplasticity and spinal automatisms, as what happens with epidural stimulation strategies. $^{29-35}$
In addition to stimulator-induced movements, neuroplasticity still occurs intensively, as demonstrated by the marked gains of patients with cervical injury and high thoracic injury on the WISCI II scale, which only captures gait. Gait training demands core control, which could not be generated by the stimulation of the sciatic, femoral, or pudendal nerves; it is very likely a result of the chronic stimulation, like what is observed in epidural stimulation strategies.

In other words, the stimulation of peripheral nerves combines the benefits of neuroplasticity observed with epidural neuromodulation with the motor benefits of FES, making these readily 

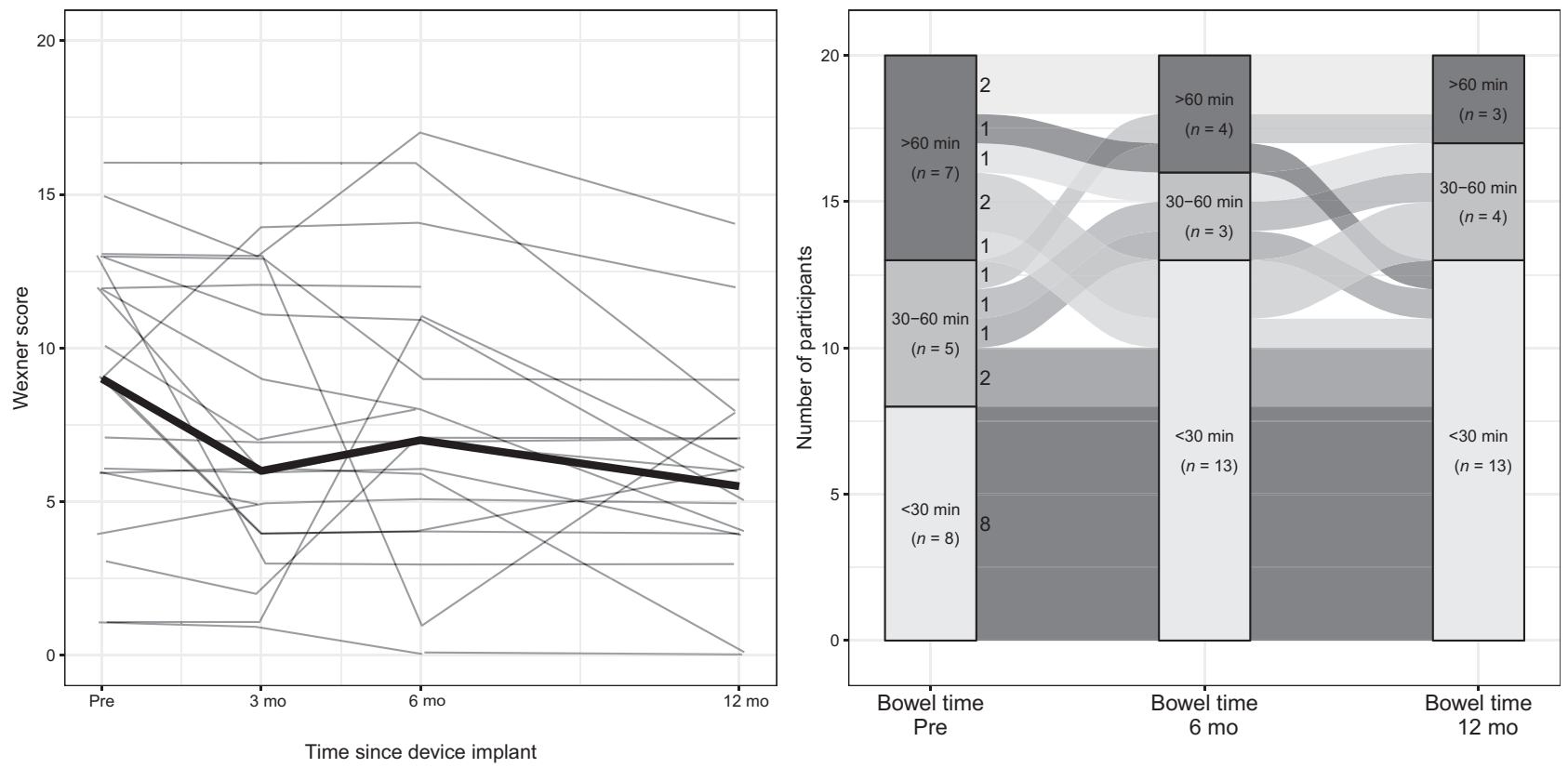

Figure 4. Bowel function. The left panel shows individual trajectories and the median of the Wexner score at four time points (presurgery and 3, 6, and 12 months after surgery). The right panel shows the distribution of patients across three groupings of the reported duration for bowel proceedings at three time points (presurgery and three and 12 months after surgery). The area of each component of a bar is proportional to the number of patients in the corresponding group (the $n$ in the bars reflects the total number of participants in each group, and the number in the shaded area reflects the number of individuals at each trajectory). Each shaded curve connecting the bars at two times corresponds to a different pattern of outcomes between the two times.

available through the pressing of a button on a remote control. Therefore, the implanted device makes it possible for patients to take advantage of the benefits of FES from the rehabilitation environment $^{37}$ to their routines, through incorporating the neuromodulator-generated movements into their ADLs.

Although the number of weekly incontinence episodes and the severity of nocturnal enuresis were significantly reduced, changes in urinary-related quality of life (Qualiveen questionnaire) or urodynamics parameters did not reach statistical significance. Analysis of these parameters was hindered, however, by the low response rate of quality-of-life questionnaires and the lack of power of our sample for these secondary outcome measures.

Female patients showed a trend for short-term reduction in sexual function, followed by a recovery to preoperative levels. Although our sample of female patients (seven in total) is underpowered for this analysis, if this drop reflects a true phenomenon, it can be explained by the limitations imposed during the first three postoperative months, aiming at reducing the risk of electrode dislocation. The trend toward improvement in male patients likely reflects a positive impact of the stimulation on erection. Moreover, about $70 \%$ of the patients (men and women) reported improvement of genital sensitivity. No definitive conclusions can be drawn from these results, however, because our sample size is underpowered to detect an MCID on the sexual outcome measures we have assessed.

Stimulation impact on anorectal function was more obvious, with statistically and clinically significant reductions on FISI and time spent to perform bowel proceedings. Although an MCID has not been established for the time to perform bowel proceedings, a reduction of -2 to -3 points is considered clinically relevant for the FISI. $^{24}$ There are a few explanations for anorectal results to be more evident than the urinary ones. Firstly, the isolated impact of sphincter and levator ani muscle function on anal continence is more important than on urinary continence, which suffers significant influence of detrusor overactivity; because pudendal stimulation directly results in increased anal sphincter and levator ani muscle tone, it acts on the main mechanism of fecal continence. ${ }^{38}$ Secondly, time to perform bowel proceedings also largely depends on core motor function, which is substantially improved, as demonstrated by mobility results, facilitating transfers to and from toilet and wheelchair, for example. One of the limitations of this study was the lack of analysis of how these objective improvements on bowel function affect their quality of life; unfortunately, up to now, there has been no translation into Brazilian Portuguese available for condition-specific questionnaires validated for neurogenic bowel dysfunction.

These results confirm the findings of Possover, ${ }^{6-10}$ who, despite the ground-breaking approach, has so far only published narrative case series. We believe that we are now reporting the largest and most well-documented case series of the Possover-LION procedure in the literature, demonstrating marked improvement on mobility of patients with chronic SCl. To our knowledge, this also is the first case series reported by a different institution and in a different country and population, demonstrating the reproducibility of the procedure outcomes.

This study has quite a few limitations, however. One of these limitations is the low response rates on questionnaires and the refusal of some patients to undergo all proposed urodynamic follow-ups. We are not allowed to ask patients about their reasons not to fill up the questionnaires, because this may be felt as coercive. It is possible that the refusals were motivated by poor results. However, the numbers of nonrespondents are consistent across all patient-reported outcomes, indicating that there is a subgroup of patients who simply do not want to answer 

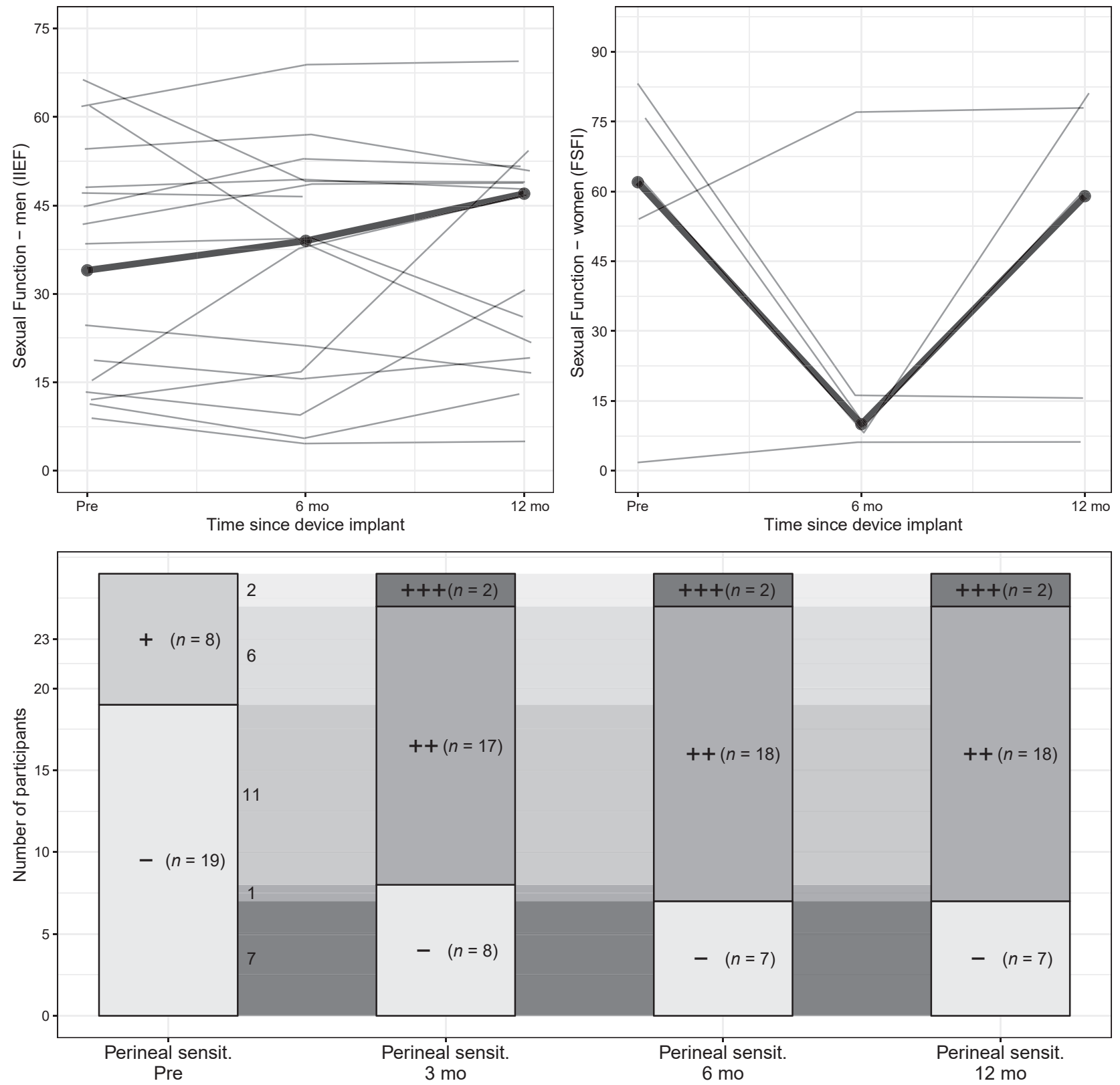

Figure 5. The top row shows individual trajectories and the median for sexual function in men (IIEF, left panel) and women (FSFI). The bottom row shows the distribution of all patients across four groupings of reported perineal sensitivity at four time points (presurgery and three, six, and 12 months after surgery). The area of each component of a bar is proportional to the number of patients in the corresponding group (the $n$ in the bars reflects the total number of participants in each group, and the number in the shaded area reflects the number of individuals at each trajectory). Each shaded curve connecting the bars at two adjacent times corresponds to a different pattern of outcomes between the two times. -, absent; +, deep/thermal sensation only; ++, deep and superficial sensation, but still subnormal; +++, normal genital sensation. Perineal sensit., perineal sensitivity.

questionnaires. If response depended on outcomes, we would expect higher response rates for questions about anorectal variables, which produced better results. Regardless of the low response rate, this study is underpowered for detecting MCIDs in most of the secondary outcome measures, as previously stated.

Another limitation is the lack of control patients. We are currently running a randomized controlled trial (RCT) on the topic, but recruitment has been particularly challenging because of patients not accepting randomization; most patients who look for our institution opt to receive the neuromodulator and do not accept the risk of being randomized into the control group. We expect to finish recruitment for our RCT in one year, with results expected in two to three years. This RCT compares the outcomes of the same rehabilitation strategy in patients with and without an implanted neuromodulator and will allow for the quantification of the overall isolated impact of neuromodulation on the rehabilitation process.

Regardless of the above limitations, there is, to our knowledge, no other treatment in the literature allowing for a $70 \%$ rate of independent walker-based gait in a population of patients with 
paraplegia with a chronic $\mathrm{SCl}$, within just one year of treatment. This finding alone makes the concept of peripheral nerve stimulation through surgically-implanted electrodes extremely attractive to the rehabilitation of this population.

These results set directions for future investigations, such as the use of brain-machine interfaces to control the neuromodulator, which will allow for complex movements generated by self-agency, consequently improving gait quality. We also are starting to investigate the impact of virtual and augmented reality (VAR) on the rehabilitation of these patients. Theoretically, neuromodulation will enhance VAR results and vice versa. We also plan to use VAR to assess the impact of improved motor function on cognitive performance. We are also analyzing the impact of neuromodulation and the independent gait on muscular fiber architecture and distribution through biopsies of the rectus femoralis muscle at the time of implantation and at one-year follow-up. We expect these biopsies to provide more elements to better understand the mechanisms of action of peripheral nerve stimulation. Potential metabolic benefits of this procedure still need to be assessed, such as its effect on bone mineral density and insulin resistance.

Finally, as the number of patients who have undergone the procedure continues to grow, we will further understand the most reliable predictors of success and the potential complications of this procedure, to better counsel our patients. Nevertheless, the results presented here are extremely encouraging.

\section{Acknowledgements}

The authors thank Priscila Souza, Maria Salete Conde, Ana Claudia Barbosa, Lisandra Machado, and Renato Moretti Marques for their volunteer work in patient care and data collection. The authors thank Vishu Chakravarti for her support on data base cleaning and preparation for statistical analysis. The authors thank Catia Mendes Pereira for manuscript formatting and submission. In memoriam of a dear friend and supporter, Frank Zietolie, CEO of Unicasa Móveis SA, who has contributed substantial funds to the execution of this study but could not see the results of his philanthropy because of his tragic early passage before the conclusion of this study. In memoriam of our dear professor, Manoel João Batista Castello Girão, who perished from complications of COVID19 during the revision process of this manuscript. May we have the wisdom and strength to carry on his good work.

\section{Authorship Statements}

This manuscript was initially drafted by Nucelio Lemos and further developed and approved by all other authors. Nucelio Lemos, Gustavo L. Fernandes, George Tomlison, Jean Faber, Acary S.B. Oliveira, and Manoel J.B.C. Girão vouch for the data and analyses and for the fidelity of this report to the study protocol and data analysis plan. Nucelio Lemos, Acary S.B. Oliveira, and Manoel J.B.C. Girão designed the trial and study protocol. Nucelio Lemos, Gustavo L. Fernandes, Augusta M. Ribeiro, Priscila S. Maia-Lemos, Wellington Contiero, and Victor Croos-Bezerra conducted the study and collected the data. Nucelio Lemos, George Tomlison, and Jean Faber verified the data and did the statistical analysis. All authors contributed to data interpretation and critical review and revision of the manuscript. Nucelio Lemos had full access to all the data in the study and had final responsibility for the decision to submit for publication.

\section{How to Cite This Article}

Lemos N., Fernandes G.L., Ribeiro A.M., Maia-Lemos P.S., Contiero W., Croos-Bezerra V., Tomlison G., Faber J., Oliveira A.S.B., Girão M.J.B.C. 2023. Rehabilitation of People With Chronic Spinal Cord Injury Using a Laparoscopically Implanted Neurostimulator: Impact on Mobility and Urinary, Anorectal, and Sexual Functions. Neuromodulation 2023; 26: 233-245.

\section{SUPPLEMENTARY DATA}

To access the supplementary material accompanying this article, visit the online version of Neuromodulation: Technology at the Neural Interface at www.neuromodulationjournal.org and at https:// doi.org/10.1016/j.neurom.2022.01.010.

\section{REFERENCES}

1. Rouanet C, Reges D, Rocha E, Gagliardi V, Silva GS. Traumatic spinal cord injury: current concepts and treatment update. Arq Neuropsiquiatr. 2017;75:387-393. https://doi.org/10.1590/0004-282X20170048.

2. Craig A, Nicholson Perry KN, Guest R, et al. Prospective study of the occurrence of psychological disorders and comorbidities after spinal cord injury. Arch Phys Med Rehabil. 2015;96:1426-1434. https://doi.org/10.1016/j.apmr.2015.02.027.

3. Craig A, Guest R, Tran Y, Middleton J. Cognitive impairment and mood states after spinal cord injury. J Neurotrauma. 2017;34:1156-1163. https://doi.org/10.1089/neu. 2016.4632.

4. Alam M, Zheng YP. Motor neuroprosthesis for injured spinal cord: who is an ideal candidate? Neural Regen Res. 2017;12:1809-1810. https://doi.org/10.4103/16735374.219041.

5. Sturma A, Hruby LA, Prahm C, Mayer JA, Aszmann OC. Rehabilitation of upper extremity nerve injuries using surface EMG biofeedback: protocols for clinical application. Front Neurosci. 2018;12:906. https://doi.org/10.3389/fnins.2018.00906.

6. Possover M. The sacral LION procedure for recovery of bladder/rectum/sexual functions in paraplegic patients after explantation of a previous Finetech-Brindley controller. J Minim Invasive Gynecol. 2009;16:98-101. https://doi.org/10.1016/j.jmig. 2008.09.623.

7. Possover M, Schurch B, Henle KP. New strategies of pelvic nerves stimulation for recovery of pelvic visceral functions and locomotion in paraplegics. Neurourol Urodyn. 2010;29:1433-1438. https://doi.org/10.1002/nau.20897.

8. Possover M. Recovery of sensory and supraspinal control of leg movement in people with chronic paraplegia: a case series. Arch Phys Med Rehabil. 2014;95:610614. https://doi.org/10.1016/j.apmr.2013.10.030.

9. Possover M. The LION procedure to the pelvic nerves for recovery of locomotion in 18 spinal cord injured peoples-a case series. Surg Technol Int. 2016;29:19-25.

10. Possover M. Ten-year experience with continuous low-frequency pelvic somatic nerves stimulation for recovery of voluntary walking in people with chronic spinal cord injury: a prospective case series of 29 consecutive patients. Arch Phys Med Rehabil. 2021;102:50-57. https://doi.org/10.1016/j.apmr.2020.09.382.

11. Possover M, Rhiem K, Chiantera V. The "Laparoscopic Neuro-Navigation"-LANN: from a functional cartography of the pelvic autonomous neurosystem to a new field of laparoscopic surgery. Minim Invasive Ther Allied Technol. 2004;13:362-367. https://doi.org/10.1080/13645700410005392.

12. Benton LA, Baker LL, Bowman BR, Waters RL. Functional Electrical Stimulation: A Practical Clinical Guide. 2nd ed. Rancho Los Amigos Rehabilitation Engineering Center; 1981.

13. Jones DA, Bigland-Ritchie B, Edwards RHT. Excitation frequency and muscle fatigue: mechanical responses during voluntary and stimulated contractions. Exp Neurol. 1979;64:401-413. https://doi.org/10.1016/0014-4886(79)90279-6.

14. Spinelli M, Malaguti S, Giardiello G, Lazzeri M, Tarantola J, Van Den Hombergh U. A new minimally invasive procedure for pudendal nerve stimulation to treat neurogenic bladder: description of the method and preliminary data. Neurourol Urodyn. 2005;24:305-309. https://doi.org/10.1002/nau.20118.

15. Peters KM, Killinger KA, Boguslawski BM, Boura JA. Chronic pudendal neuromodulation: expanding available treatment options for refractory urologic symptoms. Neurourol Urodyn. 2010;29:1267-1271. https://doi.org/10.1002/nau.20823.

16. Dittuno PL, Ditunno JF Jr. Walking index for spinal cord injury (WISCI II): scale revision. Spinal Cord. 2001;39:654-656. Published correction appears in Spinal Cord. 2009:47:349.

17. D'Ancona CA, Tamanini JT, Botega N, et al. Quality of life of neurogenic patients: translation and validation of the Portuguese version of Qualiveen. Int Urol Nephrol. 2009;41:29-33. https://doi.org/10.1007/s11255-008-9402-3. 
18. Rockwood TH, Church JM, Fleshman JW, et al. Patient and surgeon ranking of the severity of symptoms associated with fecal incontinence: the fecal incontinence severity index. Dis Colon Rectum. 1999;42:1525-1532. https://doi.org/10.1007/ BF02236199.

19. Reis AL, Reis LO, Saade RD, Santos CA Jr, de Lima ML, Fregonesi A. Validation of Portuguese version of Quality of Erection Questionnaire (QEQ) and comparison to International Index of Erectile Function (IIEF) and RAND 36-Item Health Survey. Int Braz J Urol. 2015;41:155-167. https://doi.org/10.1590/S1677-5538.IBJU.2015.01.21.

20. Thiel RRC, Dambros M, Palma PCR, Theil M, Riccetto CLZ, Ramos MF. Translation into Portuguese, cross-national adaptation and validation of the Female Sexua Function Index. Article in Portuguese. Rev Bras Ginecol Obstet. 2008;30:504-510. https://doi.org/10.1590/s0100-72032008001000005.

21. Clavien PA, Barkun J, de Oliveira ML, et al. The Clavien-Dindo classification of surgical complications: five-year experience. Ann Surg. 2009;250:187-196.

22. Scivoletto G, Tamburella F, Laurenza L, Torre M, Molinari M, Ditunno JF. Walking Index for Spinal Cord Injury version II in acute spinal cord injury: reliability and reproducibility. Spinal Cord. 2014;52:65-69. https://doi.org/10.1038/sc.2013.127.

23. Jayadevappa R, Cook R, Chhatre S. Minimal important difference to infer changes in health-related quality of life-a systematic review. J Clin Epidemiol. 2017;89:188198. https://doi.org/10.1016/j.jclinepi.2017.06.009.

24. Bols EMJ, Hendriks HJM, Berghmans LCM, Baeten CGMI, de Bie RA. Responsiveness and interpretability of incontinence severity scores and FIQL in patients with fecal incontinence: a secondary analysis from a randomized controlled trial. Int Urogynecol J. 2013;24:469-478. https://doi.org/10.1007/s00192-012-1886-9.

25. Krychman M, Rowan CG, Allan BB, Durbin S, Yacoubian A, Wilkerson D. Effect of single-session, cryogen-cooled monopolar radiofrequency therapy on sexual function in women with vaginal laxity: the VIVEVE I Trial. J Womens Health (Larchmt). 2018;27:297-304. https://doi.org/10.1089/jwh.2017.6335.

26. Rosen RC, Allen KR, Ni X, Araujo AB. Minimal clinically important differences in the erectile function domain of the International Index of Erectile Function scale. Eur Urol. 2011;60:1010-1016. https://doi.org/10.1016/j.eururo.2011.07.053.

27. Brindley GS. History of the sacral anterior root stimulator, 1969-1982. Neurourol Urodyn. 1993;12:481-483. https://doi.org/10.1002/nau.1930120506.

28. Brindley GS. Emptying the bladder by stimulating sacral ventral roots. J Physiol. 1974;237:15P-16P.

29. Gill ML, Grahn PJ, Calvert JS, et al. Neuromodulation of lumbosacral spinal networks enables independent stepping after complete paraplegia. Nat Med. 2018;24:1677-1682. https://doi.org/10.1038/s41591-018-0175-7.

30. Harkema S, Gerasimenko Y, Hodes J, et al. Effect of epidural stimulation of the lumbosacral spinal cord on voluntary movement, standing, and assisted stepping after motor complete paraplegia: a case study. Lancet. 2011;377:1938-1947. https://doi.org/10.1016/S0140-6736(11)60547-3.

31. Rejc $E$, Angeli CA, Atkinson D, Harkema SJ. Motor recovery after activity-based training with spinal cord epidural stimulation in a chronic motor complete paraplegic. Sci Rep. 2017;7:13476. https://doi.org/10.1038/s41598-017-14003-w.
32. Grahn PJ, Lavrov IA, Sayenko DG, et al. Enabling task-specific volitional motor functions via spinal cord neuromodulation in a human with paraplegia. Mayo Clin Proc. 2017;92:544-554. https://doi.org/10.1016/j.mayocp. 2017.02.014.

33. Wenger N, Moraud EM, Gandar J, et al. Spatiotemporal neuromodulation therapies engaging muscle synergies improve motor control after spinal cord injury. Nat Med. 2016;22:138-145. https://doi.org/10.1038/nm.4025.

34. Dietz V, Fouad K. Restoration of sensorimotor functions after spinal cord injury. Brain. 2014;137:654-667. https://doi.org/10.1093/brain/awt262.

35. Lu DC, Edgerton VR, Modaber $M$, et al. Engaging cervical spinal cord networks to reenable volitional control of hand function in tetraplegic patients. Neurorehabil Neural Repair. 2016;30:951-962. https://doi.org/10.1177/15459683 16644344.

36. James ND, McMahon SB, Field-Fote EC, Bradbury EJ. Neuromodulation in the restoration of function after spinal cord injury. Lancet Neurol. 2018;17:905-917. https://doi.org/10.1016/S1474-4422(18)30287-4.

37. Sadowsky $\mathrm{CL}$, Hammond ER, Strohl AB, et al. Lower extremity functional electrical stimulation cycling promotes physical and functional recovery in chronic spinal cord injury. J Spinal Cord Med. 2013;36:623-631. https://doi.org/10.1179/ 2045772313Y.0000000101.

38. Matzel KE, Schmidt RA, Tanagho EA. Neuroanatomy of the striated muscular anal continence mechanism. Implications for the use of neurostimulation. Dis Colon Rectum. 1990;33:666-673. https://doi.org/10.1007/BF02150742.

\section{COMMENT}

This is a case series of patients with SCI who had LION electrodes, documenting walking, functionality, bowel and urinary symptoms, and sexual function up to 12 months after surgery. Per the authors, this is the largest case series in the literature about this particular procedure. There is documentation of a variety of important outcomes, including walking, urinary quality of life, and sexual function, which most procedures do not study. The authors also, as they clearly pointed out in this revision, included a measure of quality of life as affected by urinary symptoms (the Qualiveen).

Kate Meriwether, MD Louisville, KY, USA 